\title{
Mobile bot: um chatterbot educacional para dispositivos móveis
}

\author{
Rony Marcolino de Andrade ${ }^{1}$
}

\begin{abstract}
Resumo: Os programas que tentam simular diálogos com o ser humano recebem o nome de robôs de conversação ou chatterbots. Ao se utilizar os chatterbots na educação é possível relacionar os alunos e o computador através da linguagem natural, por sua vez, o emprego dos chatterbots em dispositivos móveis possibilita a interação do aluno com um agente pedagógico disponível em qualquer lugar e qualquer momento. Nesse sentido, o objetivo deste trabalho é propor o desenvolvimento e avaliação do Mobile bot, um chatterbot educacional para uso em dispositivos móveis com conhecimentos sobre internet.
\end{abstract}

\section{Palavras-chave: Chatterbot. Dispositivos móveis. Robô de conversação.}

\begin{abstract}
Programs that try to simulate conversations with humans are called robot of conversation or chatterbots. When using the chatterbots in education allow the relation between the students and the computer through natural language, in turn the use of chatterbots in mobile devices enables student interaction with a pedagogical agent available anywhere and anytime. In this sense, this paper proposes the development and evaluation of Mobile bot, a educational chatterbot for use on mobile devices with knowledge about internet.
\end{abstract}

Keywords: Chatterbot. Education. Mobile devices.

\section{Introdução}

Por volta de 1950, Alan Turing propõe um jogo chamado de jogo da imitação, o qual permitiria a avaliação da capacidade dos computadores de imitar o diálogo humano. A ideia de Turing era simular um dialogo humano de modo convincente a ponto de não se distinguir a diferença entre o humano e o computador.

Nesse sentido, surgem os chatterbots, ou robôs de conversação, que são programas que tentam simular uma conversação, com o objetivo de levar o interlocutor a pensar que está falando com outro ser humano [10].

Do ponto de vista tecnológico atual, os smartphones ${ }^{2}$ possuem um perfil de aperfeiçoamento de desempenho, o que permite a implementação de uma diversidade de aplicações. O poder de processamento de áudio, imagens e vídeos, ou seja, recursos multimídias que, sem dúvida, favorecem aplicações educacionais.

O crescimento exponencial de dispositivos móveis permite aplicações em diversas áreas do cotidiano, essas aplicações quando voltadas à educação se diferenciam dos paradigmas tradicionais de ensino e aprendizagem. O aprendizado em momentos e lugares fora do que tradicionalmente conhecemos como estabelecimento de ensino, faz parte da realidade de uma cultura cada vez mais familiarizada com as tecnologias digitais.

A maioria dos chatterbots com fins educacionais está disponível na internet, nesse sentido, transportar um aplicativo como um robô de conversação para os dispositivos móveis permite que se explorem as interações pedagógicas que se diferenciam dos paradigmas tradicionais mesmo sem conectividade com a internet.

\footnotetext{
${ }^{1}$ Curso de Especialização em Tecnologias na Educação, UEPB, Campus 1 - Campina Grande (PB) - Brasil \{ronymarcolino@live.com\}

2 É um tipo de "telefone esperto" capaz de reproduzir muitas das configurações de um computador.
}

http://dx.doi.org/10.5335/rbca.2012.2440 
Sendo assim, desenvolveu-se um chatterbot para dispositivos móveis com conhecimento acerca de internet e que interaja com alunos com o intuito de simular o comportamento humano.

\section{Os chatterbots e a análise da conversação}

Em torno dos anos de 1950, Alan Turing propusera o jogo da imitação, no qual uma máquina teria o objetivo de se passar por um ser humano, esse jogo foi utilizado para se avaliar a inteligência de uma máquina e, mais tarde, caracterizou o teste de Turing.

Nesse contexto aparecem os chatterbots ou robôs de conversação, segundo Sganderla, Ferrari e Geyer [7], os chatterbots são agentes inteligentes desenvolvidos para simular uma conversa através da troca de mensagens de texto, com o intuito de tornar mais familiar a interação entre o homem e a máquina. A área que se preocupa em resolver esses tipos de desafios é a inteligência artificial, especialmente o processamento de linguagem natural (PLN).

Diversas aplicações foram desenvolvidas, desde o entretenimento até os $F A Q s^{3}$, tendo em vista a necessidade no atendimento automático a clientes. Essa foi uma das primeiras aplicações para os chatterbots. Alguns exemplos educacionais que se pode citar com os chatterbots são respectivamente o robô Elektra [8], da Universidade Federal do Rio Grande do Sul (UFRGS), destinado ao ensino de conceitos sobre redes de computadores, e o robô $\mathrm{ED}^{4}$, destinado à conscientização do público acerca da preservação de energia e de recursos naturais. Essas aplicações possuem algumas características em comum, caracterizadas justamente por serem executadas em servidor web, o que demanda uma conexão constante com a internet. No que diz respeito à conversação, o robô ED possui uma desenvoltura maior e consegue se aproximar mais de um diálogo humano.

Tendo em vista a necessidade de uma aproximação ainda maior do diálogo convincente com um robô, precisa-se delinear bem o conjunto de informações para a base de conhecimento do robô. A seguir, serão abordadas algumas nuanças presentes no comportamento da conversação que poderão ser utilizadas na afinação de um diálogo com seres humanos.

Considera-se em uma conversa um conjunto de comportamentos e estruturas que poderão existir entre os falantes de modo que um diálogo seja estabelecido. Um dos princípios básico é uma regra da conversação: fala um de cada vez, com a alternância de falantes [2].

A interação textual é uma das maneiras mais simples de se realizar um diálogo no meio digital, sendo também utilizada para realizar um diálogo com o chatterbot. Uma conversa possui estruturas e comportamentos característicos. Segundo Marcuschi [2], uma conversa geralmente é feita através de perguntas e respostas, asserções e réplicas, consistindo normalmente numa série de turnos alternados que compõem sequências em movimentos coordenados e cooperativos.

Entre essas sequências existem algumas altamente padronizadas quanto à sua estruturação, que recebem o nome de pares conversacionais, que, muitas vezes, representam uma concorrência obrigatória dificilmente adiável ou cancelável, como no caso dos cumprimentos.

Consideramos como pares conversacionais os exemplos propostos por Marcuschi [2]:

- $\quad$ pergunta - resposta;

- convite - aceitação/ recusa;

- cumprimento - cumprimento;

- $\quad$ xingamento - defesa/ revide;

- $\quad$ despedida - despedida;

- $\quad$ pedido de desculpa - perdão.

\footnotetext{
${ }^{3}$ Frequently asked questions. Em português: Perguntas mais frequentes

${ }^{4}$ http://www.ed.conpet.gov.br/br/converse.php
} 
Ao lado dos organizadores conversacionais existem alguns recursos que ordenam a conversação, tais como as aberturas e fechamentos. A estrutura mais comum numa conversação é a existência de pelo menos três seções distintas, ou seja, uma abertura, um desenvolvimento e um fechamento. A seção de abertura apresenta o contato inicial, com cumprimentos ou algo semelhante, vindo em seguida a seção do desenvolvimento dos tópicos, e finalmente a despedida ou saídas do tema geral, perfazendo a seção do fechamento.

Um desenvolvimento eficiente das etapas de abertura e fechamento em um chatterbot garante que a conversa seja realizada de maneira convincente e convidativa, pois permite que o usuário seja incentivado à interação. Sendo assim, essas características são importantes para que o robô ganhe a credibilidade do usuário.

\section{Arquitetura básica do Mobile bot}

O Mobile bot obedece à estrutura de processamento dos chatterbots convencionais que envolvem o tratamento de estímulos inseridos nele. Esses programas geralmente são dotados com os seguintes módulos: Interface com o usuário, processador de texto, motor de busca e base de dados na linguagem AIML.

Na figura 1, segundo Mikic et al. [4], é possível visualizar uma representação da arquitetura interna de um chatterbot.

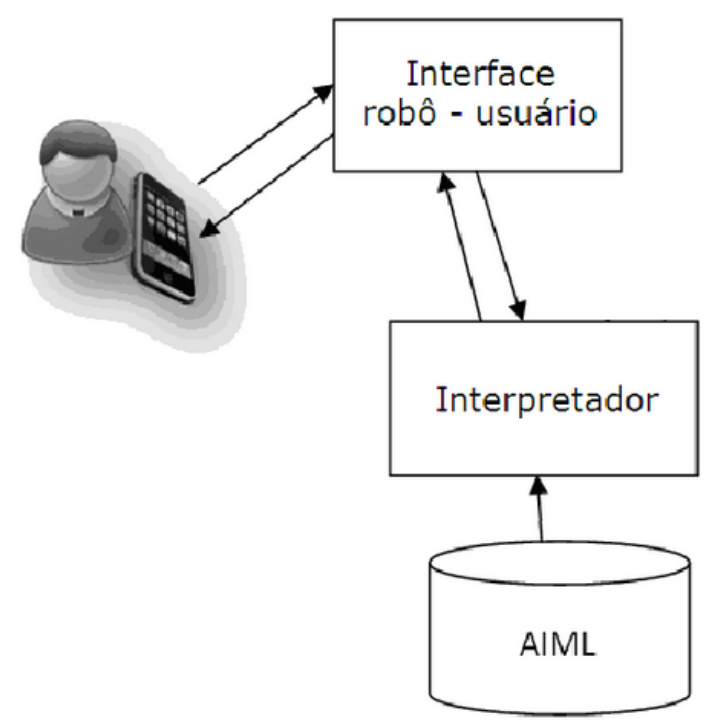

Figura 1: Arquitetura básica de um chatterbot

A interface robô-usuário permite uma interação gráfica e textual de modo a se realizar uma conversa. Nos chatterbots tradicionais o dispositivo pelo qual se tem acesso à interface é um computador conectado à internet, porém, o Mobile bot consegue estabelece um diálogo mesmo sem internet.

O interpretador tem como função adequar as informações digitadas e recebidas pelo usuário, garantindo uma busca coerente dentro do domínio de conhecimento do robô de conversação.

A base de dados AIML do chatterbot representa o domínio do conhecimento do robô e determina basicamente sobre qual assunto ele realizará o diálogo. Essa base é modelada em uma linguagem específica chamada de artificial intelligence markup language (AIML), ou seja, linguagem de marcação para inteligência artificial. A AIML é uma derivação do padrão XML ${ }^{5}$, baseada em tags.

\footnotetext{
${ }^{5}$ Extensible Markup Language - Linguagem Extensiva de Marcação.
} 
De acordo com Marçal et al.[3], a AIML permitiu a padronização e a evolução das bases de dados dos chatterbots, nesse sentido, essa padronização permite que se altere o conhecimento do robô mudando apenas o arquivo referente à sua base de dados AIML.

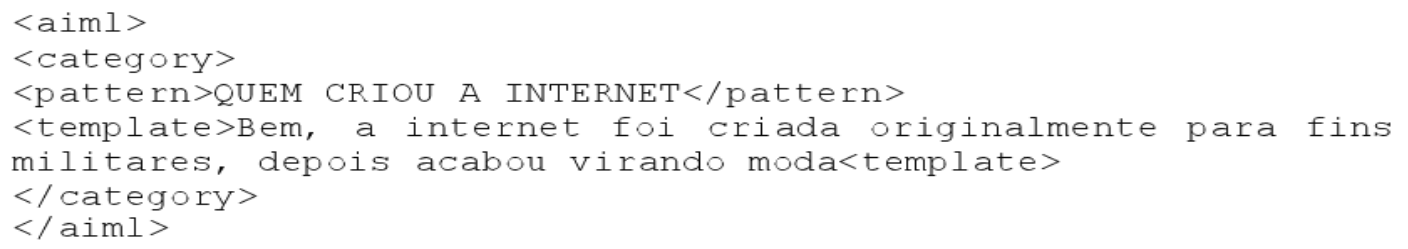

Quadro 1: Trecho e conversação, par conversacional em AIML

A AIML é orientada basicamente ao armazenamento de pares conversacionais, na qual o domínio de conhecimento do chatterbot é modelado. O Quadro 1 mostra a estrutura de um par conversacional encontrado nas bases de conhecimento AIML, composto por estímulos (patterns) e respostas (templates).

O desenho de um banco AIML deve ser criterioso, uma vez que o sistema deverá ter condições de manter o diálogo com o aluno para que assim se atinja os objetivos propostos. Foram utilizadas as informações abordadas por Ercilia e Graeff [1] para compor o banco AIML do Mobile bot.

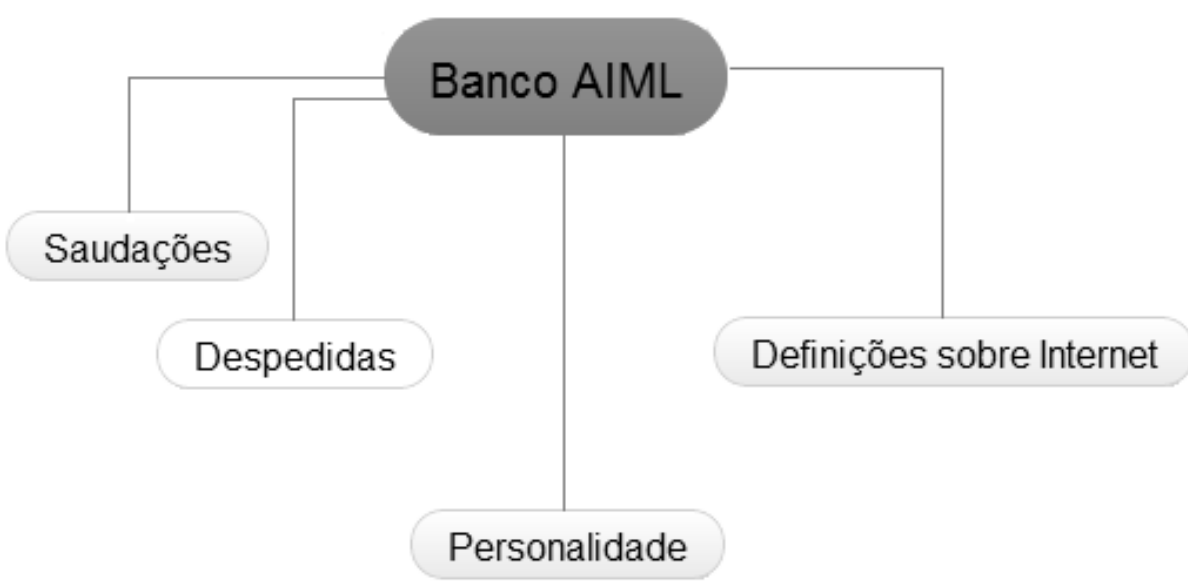

Figura 2: Mapa com a organização interna do Banco AIML

O desenho de um banco AIML deve ser criterioso, uma vez que o sistema deverá ter condição de manter o diálogo com o aluno para que assim se atinja os objetivos propostos. Foram utilizadas as informações abordadas por Ercilia e Graeff [1] para compor o banco AIML do Mobile bot.

A figura 2 representa um mapa com os conceitos existentes dentro do banco AIML, a modelagem foi feita baseado nos seguintes tópicos:

- saudações - seção destinada a uma variedade de asserções de boas vindas;

- despedidas - conjunto composto por frases de despedidas normalmente faladas;

- personalidade - em se tratando de personalidade, tem-se todas as informações que revelam algo sobre as características do próprio chatterbot, se ele é masculino, feminino ou sem sexo, se ele é jovem ou idoso. No caso, as informações inseridas caracterizam um rapaz jovem; 
- definições sobre internet - todas as informações pertinentes acerca da internet são detalhadas nessa seção.

Em se tratando das definições para compor o tópico sobre a internet, levou-se em consideração o levantamento estratégico das informações acerca da internet, desde informações como datas e países que criaram como o contexto histórico no qual ela foi criada.

\section{Chatterbots e os dispositivos móveis}

Os dispositivos móveis preenchem diversos cenários do mundo atual, sendo empregados nas mais diversas atividades. O smartphone é um dos responsáveis por oferecer aos seus usuários uma arquitetura similar aos dos computadores convencionais. Essa tecnologia favorece a exploração de um conjunto de possibilidades, como, por exemplo, a interação com multimídia através de um aparelho móvel em qualquer lugar ou qualquer momento.

Segundo a Analysis Mason ${ }^{6}$, em 2014 existirão cerca de 1.7 bilhões de smartphones ao redor do mundo. Essa quantidade de dispositivos sugere que aplicações que suportem a educação em dispositivos móveis podem se tornar uma grande oportunidade.

A educação baseada em dispositivos móveis recebe o nome de mobile learning e se caracteriza por permitir que o estudo ocorra sem que os aprendentes estejam geograficamente próximos aos professores e ainda que o aprendizado se desloque para outros contextos. Marcuschi [2] discorre sobre m-learning:

O paradigma Mobile Learning ou m-learning surge aproveitando-se da disponibilidade de dispositivos móveis e considerando-se as necessidades específicas de educação e treinamento. As pesquisas em m-Learning têm-se voltado para dois grupos de usuários principais: crianças e trabalhadores externos. Dispositivos móveis fornecem um novo e entusiasmante paradigma de interação, particularmente para as crianças [3, p. 2].

Várias iniciativas têm sido desenvolvidas nessa área, uma das mais famosas são as iniciativas de inserção de tablets e netbooks na escola. Essas iniciativas também representam uma oportunidade para o desenvolvimento de aplicações educacionais de qualidade, tendo em vista a escassez dos mesmos nos repositórios.

A melhoria dos recursos para o aprendizado do aluno é um aspecto desejável, no qual o mesmo disponha de um dispositivo computacional para execução de tarefas multimídia de forma muito mais eficiente. Em se tratando de trabalhadores externos, cujo cotidiano envolve constantes deslocamentos, a preocupação é dispor de um ambiente de aprendizado atualizado capaz de suprir as necessidades.

Em particular, dispositivos móveis oferecem uma extensão dos recursos dos computadores convencionais e permitem que algumas funcionalidades sejam aliadas à educação a distância, contribuindo para facilitar o acesso a conteúdos específicos, sem dependência de horário e de e local.

De acordo com Marçal et al. [3], é possível delinear cinco aspectos principais do mobile learning:

- expandir os limites internos e externos da sala de aula ou da empresa, de forma ubíqua, é uma reação inexorável dos efeitos da mobilidade dos dispositivos;

- permitir acesso aos recursos didáticos em qualquer lugar e a qualquer momento, de acordo com a conectividade do aparelho;

- aumentar as possibilidades de acesso ao conteúdo, promovendo a utilização dos serviços disponibilizados pelas instituições educacionais ou empresariais;

$6 \quad$ http://www.analysysmason.com/About-Us/News/Press-releases/17-billion-smartphones-by-2014-saysAnalysys-Mason 
- expandir as estratégias de ensino disponíveis, através de novas tecnologias que dão suporte tanto à aprendizagem formal como à informal;

- fornecer meios para o desenvolvimento de métodos inovadores de ensino, utilizando os recursos de computação baseados na mobilidade.

Em resumo, o m-learning surge como um potencializador do ensino e treinamento a distância. O mobile bot aparece, então, como um recurso pedagógico no qual através da conversação consegue oferecer uma metodologia compatível com as tecnologias disponíveis atualmente.

A seguir serão abordadas informações a respeito da construção do aplicativo e de como se constitui a simulação do diálogo através do dispositivo móvel mostrando como funciona o processo que resulta na condução do aluno às seções de diálogo.

\section{Aspectos da implementação do Mobile bot}

Um dos desafios das plataformas para dispositivos móveis é a sua heterogeneidade dos recursos como resoluções de tela e teclados. Isso provoca um impacto no desenvolvimento tendo em vista que os aplicativos devem funcionar na maioria dos dispositivos sem demonstrar para os usuários qualquer tipo de inadequação ou instabilidade.

Diversos tipos de dispositivos móveis, como telefones celulares, suportam aplicações na plataforma Java ME. Outro diferencial é o grande número de desenvolvedores ao redor do mundo o que fornece grandes subsídios para o desenvolvimento para os dispositivos móveis.

O programa foi desenvolvido em linguagem de programação Java para dispositivos móveis - Java ME (Micro Edition $)^{7}$. Essa linguagem é destinada aos dispositivos de pequeno porte que possuam recursos reduzidos de memória e processamento e de visualização.

Nesse projeto também foi utilizada uma Application Programming Interface (API) externa: um parser de arquivos XML, bastante eficiente e estável, que se chama $\mathrm{KXML}^{8}$. Esse parser funciona como um interpretador de arquivos junto à base de dados do robô, no qual, após realizada a leitura dos arquivos em AIML, os dados são disponibilizados para o processamento pelo chatterbot.

Abaixo segue um trecho do código referente à classe do parser responsável por extrair e organizar as seções provenientes do arquivo AIML e por sua vez retornar os dados estruturados dentro do programa.

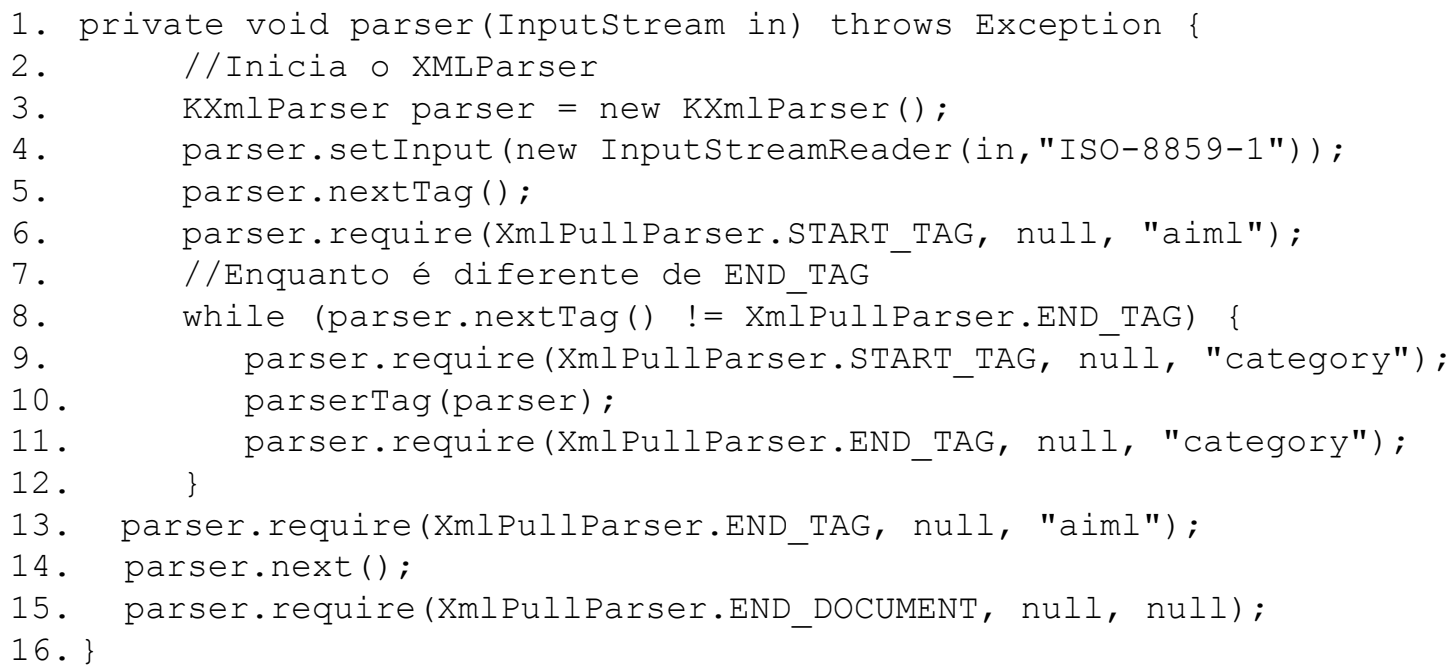

$\begin{array}{ll}7 & \mathrm{http}: / / \text { www.oracle.com/technetwork/java/javame/index.html } \\ 8 & \mathrm{http}: / / \mathrm{kxml} . \text { sourceforge.net/ }\end{array}$ 
Basicamente o funcionamento do sistema ocorre da seguinte maneira: Um texto é inserido no campo de pergunta, a qual é dividida em tokens, em seguida é submetido à avaliação, de acordo com as informações provenientes do banco de dados.

O banco é resgatado do arquivo AIML e enviado para um array de duas colunas, uma com as perguntas e outra com as respostas. Um algoritmo básico de busca procura a relação mais próxima entre o texto inserido e o presente no banco. Caso essa requisição falhe, o banco possui uma resposta padrão para que o usuário refaça a pergunta.

Para atender as necessidades iniciais da pesquisa, o Mobile bot foi criado com um banco AIML de cerca de 150 pares conversacionais, abrangendo informações de primeira instância.
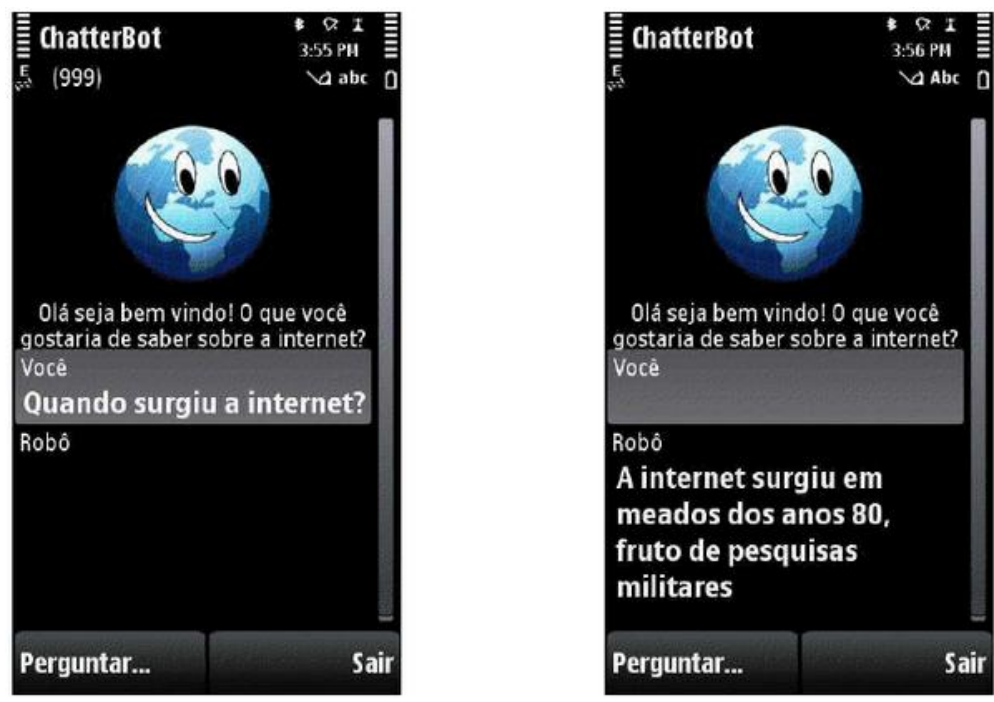

Figura 3: Telas capturadas durante a execução do chatterbot.

Na Figura 3 é possível visualizar algumas telas capturadas do chatterbot executado a partir de um smartphone. A interface do aplicativo foi criada com o objetivo de ser bastante simples e intuitiva, tendo em vista a necessidade de ser acessível e fácil para a redação do texto a ser perguntado.

Na figura 3 é possível perceber que a interface é basicamente composta por dois botões, um de "perguntar" e outro de "sair" e ainda uma ilustração de um globo antropomórfico com o intuito de representar um agente da conversa. A redação pode se realizar através da seleção direta do componente de texto, em caso de dispositivos com tela de toque, ou através das teclas de seleção em dispositivos que as possuam.

\section{Experimentos e resultados}

Nesta seção, apresentamos os experimentos realizados e os resultados obtidos após a utilização do Mobile bot nos dispositivos móveis em conjunto com os alunos. O objetivo desse experimento é avaliar influência do dispositivo móvel no ensino/aprendizagem tendo em vista a interação e o conhecimento.

Nos experimentos realizados, alunos do nível médio, técnico e superior mantiveram diálogos abertos com o Mobile bot em dispositivos como smartphones ou celulares com suporte a Java ME, realizando perguntas referentes à internet.

Os usuários tinham uma média de idade de 18 anos, possuíam familiaridade com os celulares e já haviam acessado a internet. Após os diálogos, cada usuário respondeu um formulário de avaliação, com 
questões de múltipla escolha (ruim razoável e bom), que permitiam que fossem avaliados dois critérios: (1) coerência e qualidade da conversação e (2) qualidade da interface.

Tabela 1: Telas capturadas durante a execução do Mobile bot

\begin{tabular}{l|c|c|c}
\hline Conceito avaliado & Bom & Razoável & Ruim \\
\hline $\begin{array}{l}\text { (1) Coerência e qualidade na } \\
\text { conversação }\end{array}$ & $33,3 \%$ & $60,0 \%$ & $6,7 \%$ \\
\hline (2) Qualidade da interface & $6,7 \%$ & $53,3 \%$ & $13,3 \%$ \\
\hline Total de alunos pesquisados & \multicolumn{3}{|c}{15} \\
\hline
\end{tabular}

Os resultados detectados com a avaliação dos índices apontam que a metodologia de construção da base de conhecimento proporcionou certa abrangência no conhecimento acerca das respostas dos usuários. Para as respostas que não eram entendidas o Mobile bot retornava uma mensagem padrão, a qual pedia que o usuário reformulasse o perguntado.

Apesar de não responder a algumas perguntas, a utilização do Mobile bot em sala de aula proporcionou aos alunos uma maneira interativa e simples de acessar conceitos teóricos acerca da internet, além de fomentar, entre alunos e professores, discussões que perpassam as definições básicas sobre esse assunto.

A partir dos experimentos realizados com a utilização do Mobile bot, foi possível constatar as contribuições para o ensino/aprendizagem, tanto de alunos como para professores, tais como:

a) os experimentos apontam que a utilização do Mobile bot facilita o compartilhamento de conhecimento em função do diálogo simples que se pode manter, esclarecendo dúvidas de forma prática e interativa diferente do modo tradicional de ensino;

b) possível expansão do Mobile bot para outras áreas, como eletricidade, química que envolve o aprendizado de informações estruturadas no qual o dialogo humano ajudaria na interação;

c) apoio ao ensino à distância, como o Mobile bot foi desenvolvido para dispositivos móveis é possível interagir a qualquer momento e em qualquer lugar, servindo como auxílio no aprendizado dos alunos e na prática dos professores.

\section{Considerações finais e trabalhos futuros}

Este trabalho apresentou o desenvolvimento e avaliação de um robô de conversação educacional, intitulado de Mobile bot, para dispositivos móveis, cujo objetivo é disponibilizar para os alunos e professores um agente móvel que auxilie no aprendizado e que esteja disponível em diversos ambientes fora da instituição de ensino sem que haja interação direta entre eles.

Com a inserção do Mobile bot nas atividades será possível desenvolver práticas que contribuam para o uso dos dispositivos móveis, como smartphones, e assim ele possa se transformar também em um recurso dentro de sala de aula.

Através de algumas características da inteligência artificial e do aprimoramento do hardware e do software dos dispositivos móveis é possível vislumbrar algumas possibilidades que surgem ao se explorar o potencial dessas aplicações no cenário educacional.

A conveniência e a funcionalidade oferecida pelos dispositivos permitem que muitas pessoas optem pela mobilidade. Com o crescimento do mercado, um número cada vez mais expressivo de pessoas utilizam aplicações móveis no seu cotidiano.

Esse cenário permite que os paradigmas que tanto envolvem a educação possam ser gradativamente mudados e tragam as devidas melhorias para a sociedade.

Como se esperava, as avaliações apontaram o que necessita de melhorias, tais como o aumento da fluidez e a coerência na conversação, os quais podem ser aperfeiçoados através da melhoria dos algoritmos de 
processamento do texto e com a inserção de módulos de pré-processamento, fazendo com que os Mobile bots se assemelhem aos chatterbots mais inteligentes.

Como trabalhos futuros, com base nos dados obtidos, propõe-se, ainda, o aprimoramento da interface por meio de um sistema de reconhecimento por voz, o qual permitirá que a interação ocorra de forma muito mais rápida.

Outro aspecto com o objetivo de aumentar o potencial multimídia do aplicativo é a utilização de um personagem que se apresente em formato tridimensional e possibilite uma interação mais próxima da realidade.

Com relação à plataforma utilizada, se pode sugerir ainda a programação do Mobile bot para o sistema Android, tendo em vista que essa plataforma representa um dos grandes expoentes da tecnologia móvel e possui um suporte maior para a difusão de aplicativos para dispositivos móveis. Os resultados apresentados neste artigo poderão ser vistos como ponto de partida para as próximas versões do Mobile bot.

\section{Referências}

[1] ERCILIA M.; GRAEFF A. A internet. Editora Publifolha, 2ª Edição, São Paulo. 2008.

[2] MARCUSCHI, L. A. Análise da conversação. Série princípios, Editora Ática, 1ª Edição, São Paulo. 1986.

[3] MARÇAL. E. et al. MuseuM: Uma aplicação de m-learning com realidade virtual. In: Seminário Integrado de Software e Hardware, 22. São Leopoldo: Unisinos, 2005. Disponível em: <http://www.lia.ufc.br/ great/artigos/museuM.pdf>. Acesso em: jul. 2011.

[4] MIKIC, F., A.; BURGUILLO, J.C.; LLAMAS, M.; RODRÍGUEZ, D.A.; RODRÍGUEZ, E. CHARLIE: An AIML-based chatterbot which works as an interface among INES and humans. 20th EAEEIE, 2009, Disponível em: <http://www-gti.det.uvigo.es/ darguez/pub/2009_EAEEIE_CHARLIE.pdf〉. Acesso em: jan. 2012.

[5] NEVES, M.M.A., BARROS, F.M. iAIML: Um Mecanismo para Tratamento de Intenção em Chatterbots. In ENIA - Encontro Nacional de Inteligência Artificial, Anais ... São Leopoldo: SBC, 2005.

[6] POTTER, S.A. Survey of Knowledge Acquisition from Natural Language. 1989. Disponível em: $\langle$ http://www.aiai.ed.ac.uk/project/akt/work/stephenp/TMA\%20of\%20KAfromNL.pdf $>$. Acesso em: jul. 2012.

[7] SGANDERLA, R. B.; FERRARI, D. N.; GEYER, C. F. R. BonoBOT: Um Chatterbot para Interação com Usuários em um Sistema Tutor Inteligente. In: SBIE - Simpósio Brasileiro de Informática na Educação, Anais . . . Rio de Janeiro: SBC, 2003.

[8] SCHUMAKER, R.P.; LIU, Ying.; GINSBURG, M.; CHEN, H. Evaluating mass knowledge acquisition using the ALICE chatterbot: The AZ-ALICE dialog system, 2006. Disponível em: 〈http://aim.psch.uic.edu/documents/Schumaker_etal_JHCS2006.pdf〉, Acesso em: jan. 2012.

[9] TAROUCO, L. M. R.; LEONHARDT, M. D.; CASTRO, D; DUTRA, R. "ELEKTRA: Um Chatterbot para uso em ambiente educacional" 2003.2 Disponível <http://www.cinted.ufrgs.br/renote/set2003/artigos/elektra-chatterbot.pdf>, Acesso em: Janeiro 2012.

[10] TEIXEIRA, S.; RAMIRO, T.B.; OLIVEIRA, E.; MENEZES, C.S. Chatterbots em ambientes de aprendizagem - uma proposta para a construção de bases de conhecimento. In: WIE - Workshop de Informática na Escola, Anais. . São Leopoldo: SBC, 2003. 